\title{
Pancitopenia inducida por enfermedad de Graves en un paciente con tormenta tiroidea: reporte de caso
}

\section{Graves' disease induced pancytopenia in a patient with thyroid storm: A case report}

\author{
JUAN J. MEDINA-JASSO*
}

Departamento de Endocrinología, Unidad Médica de Alta Especialidad N. ${ }^{\circ}$ 25, Instituto Mexicano del Seguro Social, Monterrey, Nuevo León, México

\section{RESUMEN}

Presentamos el caso de una mujer de 55 años de edad que acudió por tirotoxicosis, fiebre, disnea de mínimos esfuerzos y sangrado transvaginal. Su exploración física mostró exoftalmos, taquicardia, hipertermia, bocio, frote de Means-Lerman y equimosis en miembros inferiores, con puntuación de Wartofsky de 45 (tormenta tiroidea). Los exámenes de laboratorio mostraron elevación de hormonas tiroideas (tiroxina libre $4.69 \mathrm{ng} / \mathrm{ml}$ ), con hormona estimulante de la tiroides suprimida $(0.0 \mu \mathrm{UI} / \mathrm{ml})$ y pancitopenia; se descartaron otras causas de esta patología. Posterior al tratamiento con tiamazol (no lo había recibido previamente), propranolol, hidrocortisona y colestiramina se presentó mejoría de la función hematopoyética con resolución de la tormenta tiroidea.

Palabras clave: Pancitopenia. Enfermedad de Graves. Tormenta tiroidea. Tiamazol.

\begin{abstract}
We present the case of a 55-year-old woman who presented with thyrotoxicosis, fever, dyspnea with minimal effort and transvaginal bleeding. Her physical examination revealed exophthalmos, tachycardia, hyperthermia, goiter, Means-Lerman rub, and lower limb ecchymosis, with Wartofsky score of 45 (thyroid storm). Laboratory tests showed elevated thyroid hormones (free thyroxine $4.69 \mathrm{ng} / \mathrm{ml}$ ) with suppressed thyroid stimulating hormone $(0.0 \mu \mathrm{UI} / \mathrm{ml})$, and pancytopenia; other causes of this pathology were ruled out. After treatment with methimazole (she had not previously received it), propranolol, hydrocortisone, and cholestyramine, hematopoietic function improved with resolution of the thyroid storm.
\end{abstract}

Key words: Pancytopenia. Graves' disease. Thyroid storm. Methimazole.

\section{Correspondencia:}

*Juan J. Medina-Jasso

E-mail: drjuanmeja@gmail.com
Fecha de recepción: 28-06-2020

Fecha de aceptación: 20-08-2020

DOI: 10.24875/RME.20000073
Disponible en internet: 10-08-2021 Rev Mex Endocrinol Metab Nutr. 2021;8:154-7

2462-4144 / @ 2020 Sociedad Mexicana de Nutricion y Endocrinologia, AC. Publicado por Permanyer. Este es un artículo open access bajo la licencia CC BY-NC-ND (http://creativecommons.org/licenses/by-nc-nd/4.0/). 


\section{INTRODUCCIÓN}

La enfermedad de Graves es la causa de hasta el $80 \%$ de los casos de hipertiroidismo y se estima que el $0.5 \%$ de la población padece esta enfermedad. Aunque usualmente se manifiesta por síntomas de tirotoxicosis como pérdida de peso, ansiedad, palpitaciones, diarrea y temblor; en algunas ocasiones pueden existir presentaciones menos comunes (atípicas) como parálisis periódica y psicosis ${ }^{1}$; incluso la tirotoxicosis con la autoinmunidad subyacente pueden afectar el sistema hematológico ${ }^{2}$, manifestándose como citopenias (más comúnmente anemia) ${ }^{1}$; sin embargo, en raras ocasiones se ha reportado pancitopenia ${ }^{3}$, lo que puede ocasionar un dilema terapéutico en el tratamiento, ya que el uso de tiamazol puede inducir efectos hematológicos secundarios ${ }^{4,5}$.

\section{OBJETIVO}

Describir un caso de pancitopenia provocada por enfermedad de Graves en un paciente con tormenta tiroidea sin uso previo de tiamazol. Describir la respuesta hematológica al tratamiento con esteroides y tiamazol.

\section{EXPOSICIÓN DEL CASO}

Acudió al servicio de urgencias una mujer de 55 años de edad por presentar en los seis meses previos pérdida de peso de $20 \mathrm{~kg}$, diarrea, ansiedad, insomnio y palpitaciones; cinco días antes de su ingreso se agregó fiebre no registrada termométricamente, disnea de mínimos esfuerzos y sangrado transvaginal. Su exploración física mostró taquicardia de $138 \mathrm{Ipm}$, febrícula $\left(37.9^{\circ}\right)$ y temblor fino distal. En la región cervical se encontró tiroides de $30 \mathrm{~g}$, con bordes irregulares y frote de Means-Lerman en región esternal superior. En extremidades inferiores se observó mixedema y múltiples petequias y equimosis; se encontró además hemorragia transvaginal activa. Se documentó puntuación de Wartofsky de 45 consistente con tormenta tiroidea. Sus exámenes mostraron elevación de
Tabla 1. Hallazgos de laboratorio

\begin{tabular}{|c|c|c|}
\hline Parámetros & $\begin{array}{c}\text { Al ingreso } \\
\text { (sin tiamazol) }\end{array}$ & $\begin{array}{l}\text { Postratamiento } \\
\text { (20 días después) }\end{array}$ \\
\hline $\mathrm{TSH} \mu \mathrm{UI} / \mathrm{ml}$ & 0.00 & 0.00 \\
\hline T4L (ng/ml) & 4.69 & 0.99 \\
\hline T4T mg/dl & 19.13 & 10.33 \\
\hline Leucocitos/ $\mu$ l & 2,702 & 6,094 \\
\hline Neutrófilos/ $\mu$ l & 700 & 1,900 \\
\hline Linfocitos/ $\mu$ l & 1,400 & 2,200 \\
\hline Monocitos/ $\mu$ l & 300 & 300 \\
\hline $\mathrm{Hb}(\mathrm{g} / \mathrm{dl})$ & 9.1 & 11.2 \\
\hline Plaquetas/ $\mu$ l & 66,200 & 138,700 \\
\hline
\end{tabular}

TSH: hormona estimulante de la tiroides; T4L: tiroxina libre; T4T: tiroxina total; $\mathrm{Hb}$ : hemoglobina.

hormonas tiroideas, además de pancitopenia (Tabla 1); descartándose otras causas de esta alteración hematológica: el aspirado de medula ósea mostró infiltración linfocítica, normocelular; el ELISA (enzimoinmunoanálisis de adsorción) para $\mathrm{VIH}$, la prueba de VDRL (venereal disease research laboratory), anticuerpos contra citomegalovirus, toxoplasma y rubeola se reportaron negativos. El ultrasonido tiroideo mostró bocio difuso en presencia de aumento de flujo Doppler generalizado en patrón de infierno tiroideo (Fig. 1).

Posterior a tratamiento con tiamazol (dosis inicial de $80 \mathrm{mg} /$ día), propranolol (inicialmente $60 \mathrm{mg}$ cada $6 \mathrm{~h}$ ), hidrocortisona (dosis inicial de $100 \mathrm{mg}$ IV cada $8 \mathrm{~h}$ ) y colestiramina ( $4 \mathrm{~g}$ cada 6 h) se presentó aumento progresivo de líneas celulares (Tabla 1) con resolución de la tormenta tiroidea (puntuación de Wartofsky de 5). Se egresó por mejoría para tratamiento posterior con radioyodo.

\section{CONCLUSIONES}

La pancitopenia se puede asociar a una amplia variedad de condiciones como son alteraciones en la médula ósea (falla primaria, lesiones ocupantes de espacio, insuficiente producción), destrucción periférica de las células sanguíneas, infecciones o medicamentos ${ }^{6}$; incluso en raras ocasiones se ha reportado ligada al hipertiroidismo. La mayoría de las causas de pancitopenia en enfermedad de Graves son debidas a 


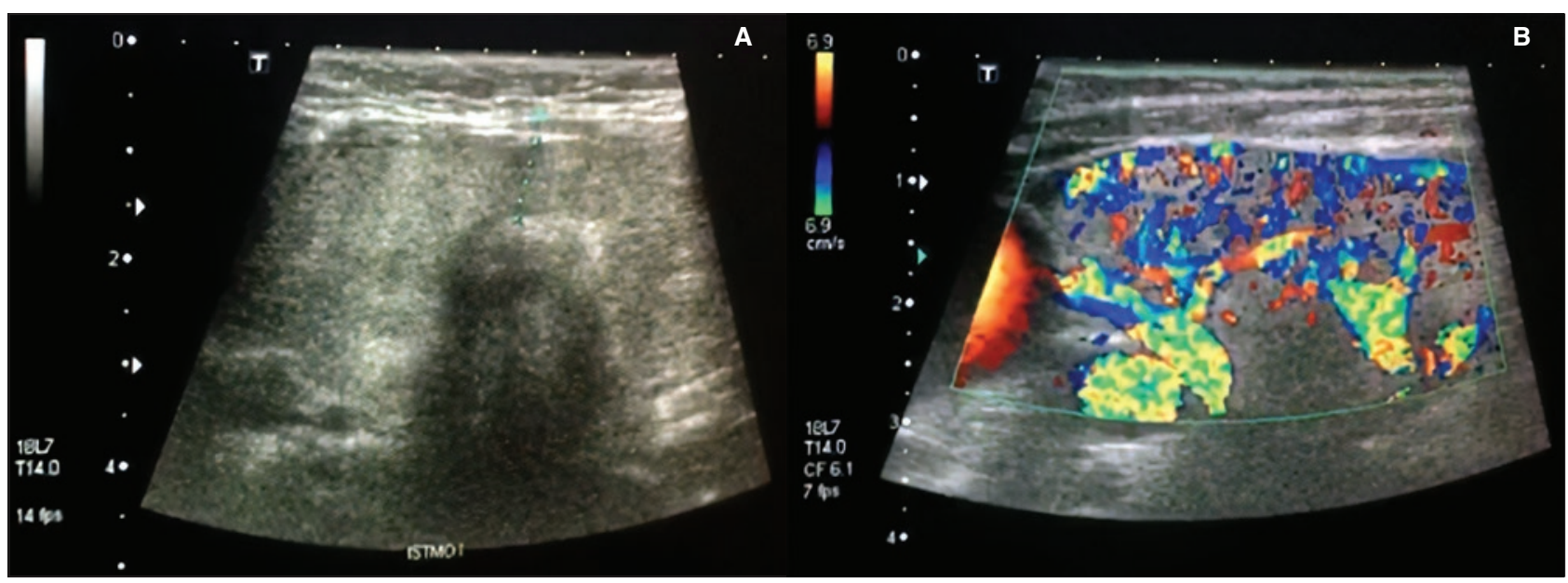

Figura 1. Ultrasonido tiroideo. Se reporta el lóbulo tiroideo izquierdo de $7.6 \times 3 \times 3.8 \mathrm{~cm}$, istmo de $0.75 \mathrm{~cm}$, y el lóbulo tiroideo derecho de $7.2 \times 2.8 \times 3.4 \mathrm{~cm}$ con un volumen total de glándula aumentado de $80.6 \mathrm{ml}$ (A). Se observa gran aumento del flujo Doppler en toda la glándula (B); lo anterior consistente con datos ecográficos de enfermedad de Graves.

complicaciones del uso de tiamazol, reportándose una prevalencia de agranulocitosis del $0.1-0.5 \%$, la cual usualmente ocurre dentro de los primeros dos a tres meses de tratamiento con un tiempo promedio de inicio a los 69 días $^{7}$. La American Thyroid Association recomienda que antes de iniciar tratamiento con fármacos antitiroideos se realice una biometría hemática y en caso de que la cuenta de neutrófilos sea menor de $1,000 / \mathrm{mm}^{3}$ se debe reconsiderar seriamente iniciar tiamazol (recomendación débil, baja calidad de evidencia) $)^{8}$; sin embargo existen escasos reportes de casos de pacientes con enfermedad de Graves y pancitopenia en los que se detecta la alteración hematológica al inicio del diagnóstico sin haber recibido tratamiento previo y que mejoran con el uso de tionamidas ${ }^{4}$. En el presente caso clínico se demostró en una paciente con enfermedad de Graves de reciente diagnóstico que el hipertiroidismo puede ocasionar en el sistema hematopoyético una complicación rara y potencialmente catastrófica: la pancitopenia. Se descartaron causas secundarias de esta y a su diagnóstico aún no se había indicado tiamazol, por lo que se consideró que esta alteración fue consecuencia directa de la concentración excesiva de hormonas tiroideas en la circulación. Los mecanismos sugeridos para esta alteración hematológica son: a) efecto directo de las hormonas tiroideas en la médula ósea causando un disturbio en la maduración y diferenciación de la célula madre hematopoyética pluripotencial ${ }^{9}$, b) hiperesplenismo asociado a la tirotoxicosis, el cual puede reducir la vida media de las células sanguíneas y c) coexistencia de anticuerpos antineutrófilos y antiplaquetas ${ }^{10}$.

Afortunadamente se demostró en este caso que el tratamiento con tiamazol e hidrocortisona remitió la tormenta tiroidea y restauró la función hematopoyética, por lo que se sugiere que:

- En todo paciente con enfermedad de Graves se debe realizar una valoración hematológica previo al inicio de tiamazol.

- La presencia de pancitopenia detectada al diagnóstico de la enfermedad de Graves y sin haber recibido terapia previa con tiamazol no se debe considerar una contraindicación para administrar dicho fármaco.

\section{AGRADECIMIENTOS}

A todos los pacientes, que nos permiten aprender y perfeccionar la labor de ser médico.

\section{FINANCIAMIENTO}

Los recursos para la realización de los estudios de laboratorio y de imagen referidos en el presente 
escrito se obtuvieron del Instituto Mexicano del Seguro Social, Unidad Médica de Alta Especialidad N. ${ }^{\circ} 25$.

\section{CONFLICTO DE INTERESES}

El autor señala que no existe ninguna relación financiera o personal con otras personas u organizaciones que pudieran dar lugar a un conflicto de intereses en relación con el presente escrito.

\section{RESPONSABILIDADES ÉTICAS}

Protección de personas y animales. Los autores declaran que para esta investigación no se han realizado experimentos en seres humanos ni en animales.

Confidencialidad de los datos. Los autores declaran que han seguido los protocolos de su centro de trabajo sobre la publicación de datos de pacientes.
Derecho a la privacidad y consentimiento informado. Los autores han obtenido el consentimiento informado de los pacientes y/o sujetos referidos en el artículo.

\section{BIBLIOGRAFÍA}

1. Hegazi MO, Ahmed S. Atypical clinical manifestations of Graves' disease: an analysis in depth. J Thyroid Res. 2012;2012:768019.

2. Ford H, Carter J. The haematology of hyperthyroidism: abnormalities of erythrocytes, leucocytes, thrombocytes and haemostasis. Postgrad Med J. 1988;64:735-42.

3. Garcia J, Silveira B, de Franca L, Wolff M, Torrini R, Ellinger V, et al. Marrow hipoplasia: a rare complication of untreated Grave's disease. Arq Bras Endocrinol Metab. 2014;58(9):953-57.

4. Lima C, Zantut D, Castro V, Tambascia M, Lorand I, Saad S, et al. Pancytopenia in untreated patients with Graves' disease. Thyroid. 2006;16(4):403-9.

5. Brent G. Graves' disease. N Engl J Med. 2008;358(24):2594-605.

6. Weinzierl E, Arber D. The differential diagnosis and bone marrow evaluation of new-onset pancytopenia. Am J Clin Pathol. 2013;139(1):9-29.

7. Watanabe N, Narimatsu H, Noh J, Yamaguchi T, Kobayashi K, Kami M, et al. Antithyroid drug-induced hematopoietic damage: a retrospective cohort study of agranulocytosis and pancytopenia involving 50,385 patients with Graves' disease. J Clin Endocrinol Metab. 2012;97(1):49-53.

8. Ross DS, Burch HB, Cooper DS, Greenlee MC, Laurberg P, Maia AL, et al. 2016 American Thyroid Association Guidelines for Diagnosis and Management of Hyperthyroidism and Other Causes of Thyrotoxicosis. Thyroid. 2016;26(10):1343-421.

9. Gianoukakis A, Leigh M, Richards P, Christenson P, Hakimian A, Fu P, et al. Characterization of the anaemia associated with Graves' disease. Clin Endocrinol. 2009;70(5):781-7.

10. Chaar B, Kudva G, Olsen T, Silverberg A, Grossman B. Thrombotic thrombocytopenic purpura and Graves' disease. Am J Med Sci. 2007;334(2):133-5. 